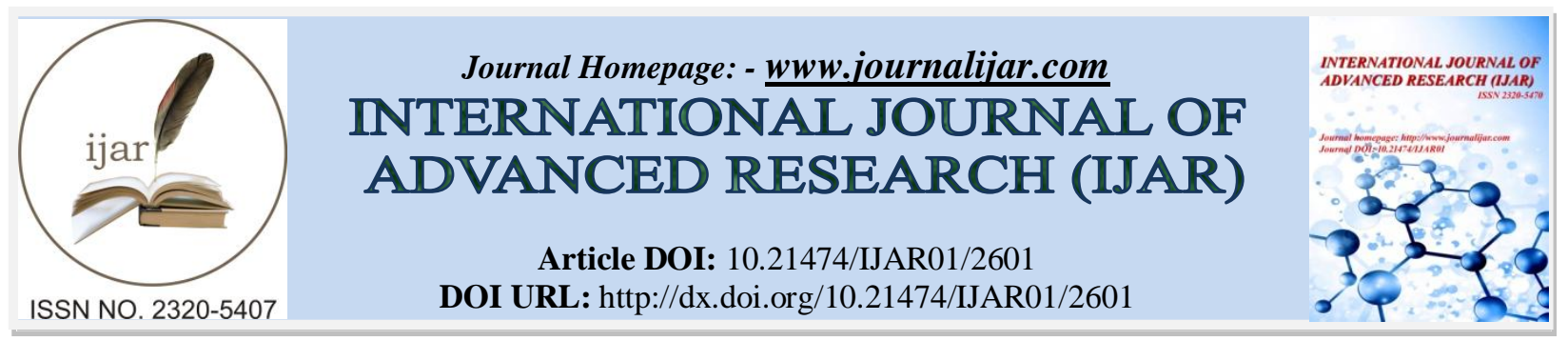

RESEARCH ARTICLE

\title{
SECURED ONLINE PAYMENT SYSTEM-A REVIEW ON VARIOUS TECHNIQUES.
}

Swapna B Sasi ${ }^{1}$, Aparna Mohan ${ }^{2}$ and Dilsha Hyderali ${ }^{2}$.

1. Asst. Professor, Deptartment of Computer Science and Engineering, Jyothi Engineering College,Kerala,India.

2. B.Tech Final Year Students, Deptartment of Computer Science and Engineering, Jyothi Engineering College,Kerala,India.

\section{Manuscript Info}

.........................

\section{Manuscript History}

Received: 28 October 2016

Final Accepted: 27 November 2016

Published: December 2016

\section{Key words:-}

Steganography, Visual Cryptography, Optical Character Recognition, Certified Authority

\section{Abstract}

Online payment system allows money transfers to be made only through Internet instead of using cash or a check, in person or by mail. Now days there is a tremendous growth in online payment throughout the world so the E-shopping users are facing more problems. The main problem is security. We provide secured online payment system through various techniques. Main techniques used are Steganography, Visual Cryptography and OCR. This paper compares various work done by different authors in the field of online payment system.

Copy Right, IJAR, 2016,. All rights reserved.

\section{Introduction:-}

In this busy world most of us prefer doing online shopping and online payment. Since there is no waste of time in this process a tremendous growth in online payment has been seen throughout the world. So, the attackers mainly focus on this process and e-shopping users are facing more problem. Security is the main problem behind this system.

Online payment is an electronic payment for goods or services electronically without using cash or check in person or by mail. It could be achieved through different modes. Different modes are Debit card, Credit card, E-money and Electronic fund transfer. Since there is less security by using this type of payment. We provide a secure payment by using different technologies.

Steganography is a data hiding technique used for hiding secret messages. The messages could be hidden under image, audio, video, text. Visual Cryptography is an encryption technique used to generate shares of images. $(2,2)$ Visual Cryptography work same as visual cryptography scheme in which 2 meaning full shares are generated. Optical character recognition also known as OCR is used to convert the typed, printed or handwritten text into encoded machine text. It can be used in machine translation, text-to-speech and text mining.

User authentication information is stored in the image first. Then the password is hidden inside the cover image using the technique known as Steganography. Snapshot of these images is taken. Next shares are generated using Visual Cryptography scheme. One share is given to customer and other share is stored in the database of certified authority. During online shopping the customer selects the object and add to cart, preferred payment directs the customer to CA port. In the port Customer submits its own share and merchant submits account details. CA combines both shares. The cover text and account details are sent to bank. From this password is recovered from the

Corresponding Author:- Swapna B Sasi

Address:- Asst. Professor, Deptartment of Computer Science and Engineering, Jyothi Engineering College,Kerala,India. 
cover text. Bank matches it with the password stored in the database and by verifying it the fund is transferred into the merchants account. Otherwise the transaction fails.

\section{Literature survey:-}

[1] Proposes a combined version of Text based steganography and visual cryptography. In the proposed text based steganography periphrases are used for hiding data rather than properties of sentence. Account number in connection with the merchant is placed over the cover text and snapshot is taken. Using Visual Cryptography shares are generated.

Steganographic algorithm[2] used to hide the text inside an image. The algorithm proposed in this paper is LSB algorithm. Instead of hiding the data in the least significant bit of RGB component the data is first converted into ASCII format and corresponding binary numbers are generated. These binary numbers are then stored in RGB component and stego image is generated.

[3] Explains about a formal method for modeling and verifying malicious behaviour in online shopping business based on petrinets. Software designs can be secured from malicious attack at process design time. This approach reduces the difficulty and cost of modification for imperfect system at release phase.

E-shopping users are facing more problem in their day to today life. The mains problems are identity theft and spear phishing attack. [4] address those problems. The author proposes combined use of Hierarchical Visual Cryptography and keyless image steganography technique for addressing those issues. HVCS is used for generating image shares and steganography is used for hiding the users details under the image share.

A large amount of e-commerce, social networking is emerging throughout the world and they are generating a large amount of data. These data's are shared between them mainly using cloud. Paper[5] represents secure data transmission in e-commerce, Social networking etc.

In [6] author explains about the enhanced least significant bit algorithm for steganography. In this algorithm a cover image is selected as input. The message to be hidden is stored only in blue component. Pixel selection filter technique is used to obtain the best area to hide the message in cover image. ELSB is applied to every pixel to hide the message then the message is hidden using bit replacement technique.

Optical Character Recognition(OCR) is used for electronic translation of handwritten, printed text into machine translated image[7]. This Paper presents an experiment on three applications such as Captcha, Institutional Repository and Optical Music Character Recognition.

In [8] the author Proposes Visual Cryptography For Print and Scan Application. A problem is faced in the precise alignment of Print and Scan Visual Cryptography Shares. A Wlash Transform is used to embed mark in both shares to get an correct alignment of these shares.

Color visual cryptography scheme[9] encrypts informative color image in such a way that result of encryption is in the form of shares. Information is scrambled in the shares hence shares do not reflect an information directly. Color visual cryptography uses 2 out of two secret sharing scheme .

Visual Cryptography Scheme Based On Pixel Expansion for Black \& White Image. The scheme explained in this paper is based on $\mathrm{k}$ out of $\mathrm{k}$ visual cryptography[10]. To extract the secret message both the shares are needed to superimpose one on another.

The design of two stage binary image security scheme is achieved using (2,2)Visual Cryptography Technique. At the first decoding stage we are not able to get back the original image. This method aims to improve efficiency of Visual cryptography scheme[11].

[12] Defines two approaches of substitution technique of audio steganography. This paper provides security by using the RSA algorithm which is based on cryptography for more secure communications. By using these methods others cannot percept the existence of message embedded in the audio file. 
Optical Character Recognition (OCR) system. It is used for camera captured image/graphics embedded textual documents for handheld devices. Because of the computing constraints of handheld devices, the author kept their study limited to light-weight and computationally efficient techniques.[13] proposes this method.

In Multiple cryptography the data will be encrypted into a cipher and the cipher will be hidden into a multimedia image file in encrypted format. Visual steganography algorithms is used to hide the encrypted data. This paper use traditional cryptographic techniques to achieve data encryption[14].

In [15] says about OCR system for offline handwritten character recognition. This system offers an upper edge by having an advantage i.e. its scalability.

Signature of the applicant is processed in such a way that, signature is taken as input and is divided into different number of shares depending upon the banks scheme. The algorithm mainly deals with Image Processing and Visual Cryptography. [16] proposes an algorithm for secured bank authentication.

\section{Conclusion:-}

Online payment system allows money transfers to be made only through Internet instead of using cash or a check, in person or by mail. Steganography, Visual cryptography are the basic techniques used for secured online payment system. This paper presents a review of these techniques. Various papers have been reviewed on Steganography, Visual cryptography and OCR. It is studied that there is various types of steganography like text, audio, image steganography. And various types of visual cryptographic schemes. Techniques like steganography,visual cryptography and OCR have been reviewed and studied in the paper.

\section{References:-}

1. Souvik Roy, P. Venkateswaran "Online Payment System Using Steganography and Visual Cryptography," 2014 IEEE Students' Conference on Electrical, Electronics and Computer Science.

2. Sharma et al., "A New Approach to Hide Text in Images Using Steganography," International Journal of Advanced Research in Computer Science and Software Engineering 3(4), April - 2013, pp. 701-708.

3. Wang Yang Yu, Chun Gang Yan "Modeling and verification of online shopping business processes by considering malicious behaviour patterns," IEEE Transactions, 2014.

4. K.S.Suganya, K.Manikandan"Enhanced Secure E-Gateway using Hierarchical Visual Cryptography," IJETT vol.3 Jan 2015- ISSN: 2349 - 9303.

5. Shyam Nandan Kumar "Cryptography during Data Sharing and Accessing Over Cloud," International Transaction of Electrical and Computer Engineers System, 2015, Vol. 3, No. 1, 12-18.

6. Shilpa Gupta1,Geeta Gujral2,Neha Aggarwal3 "Enhanced Least Significant Bit algorithm For Image Steganography," IJCEM International Journal of Computational Engineering \& Management, Vol. 15 Issue 4, July 2012.

7. Amarjot Singh, Ketan Bacchuwar, Akshay Bhasin "A Survey of OCR Applications," International Journal of Machine Learning and Computing, Vol. 2, No. 3, June 2012.

8. Wei-Qi Yan, Duo Jin, Mohan S Kankanhalli "Visual Cryptography for Print and Scan Applications," Circuits and Systems, 2004. ISCAS '04. Proceedings of the 2004 International Symposium on

9. Pallavi Vijay Chavan, R.S. Mangrulkar, Third International Conference on Emerging Trends in Engineering and technology, "Encrypting Informative Color Image using Color Visual Cryptography" 2010 IEEEDOI 10.1109/ICETET.2010.94.

10. Lekhika Chettri, "Visual Cryptography Scheme Based On Pixel Expansion for Black \& White Image", International Journal Of Computer Science and Information Technologies, Vol.5(3),2014,4190-4193.

11. Mr Rohith S, Mr Vinay G "A Novel Two Stage Binary Image Security using (2,2) Visual Cryptography Scheme," International Journal Computational Engineering Research, ISSN:2250-3005.

12. Arfan Shaikh1, Kirankumar Solanki2, Vishal Uttekar3, Neeraj Vishwakarma4" "Audio Steganography And Security Using Cryptography" International Journal of Emerging Technology and Advanced Engineering, ISO 9001:2008 Certified Journal, Volume 4, Issue 2, February 2014.

13. Ayatullah Faruk Mollah, Nabamita Majumder, Subhadip Basu, Mita Nasipuri,"Design of an Optical Character Recognition System for Camera-based Handheld Devices", International journal of Computer Science Issues, Vol. 8, Issue 4, pp. 283-289, July 2011. 
14. Neha Chhabra," Visual Cryptographic Steganography in Images", IJCSNS International Journal of Computer Science and Network Security, VOL.12 No.4, April 2012.

15. Shalin A. Chopra1, Amit A. Ghadge2, Onkar A. Padwal3, Karan S. Punjabi4, Prof. Gandhali S. Gurjar." Optical Character Recognition", International Journal of Advanced Research in Computer and Communication Engineering Vol. 3, Issue 1, January 2014.

16. Chetana Hegde, S. Manu, P. Deepa Shenoy, K. R. Venugopal, L MPatnaik, "Secure Authentication using Image Processing and Visual Cryptography for Banking Applications," Proceedings of $16^{\text {th }}$ International Conference on Advanced Computing andCommunications, pp. 65-72,Chennai, India, 2008. 